\title{
Efeito da Idade da Vaca ao Parto e da Data Juliana de Nascimento sobre Características Pré-desmama de Bezerros da Raça Gir ${ }^{1}$
}

\author{
Luciele Cristina Pelicioni², 3, 4, Lillian Pascoa2, 5, Carolina Amália de Souza Dantas Muniz², \\ Sandra Aidar de Queiroz ${ }^{2,6}$
}

\begin{abstract}
RESUMO - Objetivou-se com este trabalho estimar a influência da idade da vaca ao parto (IDV) e da data juliana de nascimento (DJN) sobre o peso à desmama (PD) e a média do ganho diário no período pré-desmama (GMD) de bezerros Gir, determinando fatores de correção para estes efeitos. Foram analisados 10.685 e 18.339 dados de PD e GMD de bezerros Gir, provenientes do Arquivo da Associação Brasileira dos Criadores de Zebu (ABCZ), pertencentes a 1229 e 1979 grupos contemporâneos (GC), respectivamente. PD e GMD foram pré-ajustados para o efeito da idade do bezerro à desmama. O efeito de IDV sobre PD e GMD foi modelado como polinômio segmentado quadráticoquadrático-quadrático, com "nós", ou pontos de junção aos 4,1; 12,7 e 4,0; 8,2 anos, respectivamente, para machos e como polinômio segmentado quadrático-quadrático, com "nó", ou ponto de junção aos 3,8 anos, para fêmeas sobre as duas características. A DJN foi modelada como um polinômio segmentado quadrático-quadrático com "nó" aos 126 dias para PD e 167 dias para GMD. Os resultados mostraram que a determinação dos fatores de correção para IDV deve ser feita, separadamente, para machos e fêmeas e, para DJN, deve-se considerar cada estação do ano, para que as diferenças entre elas sejam bem observadas. Os fatores de correção para o efeito da idade da vaca variaram de 0,94750 a 1,08033 sobre PD e 0,91714 a 1,07689 sobre GMD, para machos, e de 0,90937 a 1,07415 sobre PD e 0,96055 a 1,14007 sobre GMD, para fêmeas. Para o efeito de DJN, a amplitude foi de 0,9256 a 1,0340 sobre PD e 0,9112 a 1,0551 sobre GMD.
\end{abstract}

Palavras-chave: fatores de correção, ganho médio diário pré-desmama, peso à desmama, polinômio segmentado

\section{Effect of Age of Dam at Calving and Julian Date of Birth on Pre-Weaning Traits of Gyr Calves}

\begin{abstract}
The aims of this research were to estimate the influence of age of dam at calving (AC), and julian date of birth (JDB) on weaning weight (WW) and preweaning average daily gain (ADG) of gyr calves and adjustment factors for them. It was analyzed 10,685 and 18,339 records, on WW and ADG of Gyr calves, from the Associação Brasileira dos Criadores de Zebu data set belonging to 1229 and 1979 contemporary groups (CG), respectively. AC, for males, was fitted as a segmented polynomial quadratic-quadratic-quadratic with stage changing at 4.1, 12.7 for WW and 4.0, 8.2 years old for ADG, and as a segmented polynomial quadratic-quadratic with state changing at 3.8 years old for female. JDB was fitted as a segmented polynomial quadratic-quadratic with state changing at 126 days for WW and 167 days for ADG. The results showed that the adjustment factors for AC must be done separately, for males and females and for JDB, each season must be considered, so that the differences among them can be better observed. The adjustment factors to $\mathrm{AC}$, for males, ranged from 0.94750 to 1.08033 for WW and 0.991714 to 1.07916 for ADG and, for females from 0.90937 to 1.07415 for WW and 0.96055 to 1.14007 for ADG. The adjustment factors to JDB ranged from 0.9256 to 1.0340 for WW and 0.9112 to 1.0551 for ADG.
\end{abstract}

Key Words: adjustment factors, Gyr cattle, preweaning average daily gain, segmented polynomial, weaning weight

\section{Introdução}

Os índices reprodutivos e produtivos da pecuária de corte brasileira encontram-se muito aquém do desejável, principalmente, em raças que se desenvolveram e estão sendo utilizadas para um duplo propósito: produção de carne e de leite, como é o caso da raça Gir.
A competitividade, imposta pelo mercado, exige um bovino adequado a um sistema de produção de ciclo curto, o mais adaptado possível às condições tropicais. Dessa forma, busca-se otimizar o tempo necessário para o animal atingir a idade do abate.

Do nascimento à desmama, vários são os fatores que influem no desempenho dos animais. No caso dos

\footnotetext{
${ }^{1}$ Parte do trabalho de Graduação do segundo autor como parte das exigências para a obtenção do grau de Zootecnista - FCAV - UNESP Jaboticabal, SP.

2 Departamento de Zootecnia, MGA - FCAV - UNESP - CEP: 14.884-900 - Jaboticabal, SP.

3 Aluna de Doutorado do Programa de Pós-Graduação em Zootecnia - Produção Animal - FCAV - UNESP.

${ }^{4}$ Bolsista FAPESP. E.mail: lucripe@fcav.unesp.br

5 E.mail: Ilpascoa@ata.terra.com.br

6 Bolsista CNPq. E.mail: saquei@fcav.unesp.br
} 
bovinos de corte, os efeitos ambientais importantes para o peso ao desmame e ganho de peso no período do nascimento ao desmame que, normalmente, não são incluídos no grupo contemporâneo e que podem ser pré-ajustados, são: idade do bezerro ao desmame (IDB), idade da vaca (IDV) e data de nascimento dentro do calendário juliano (DJN).

Estes efeitos, de origem ambiental, são facilmente controlados estatisticamente pelas médias de fatores de ajustamento, com o objetivo de se ajustarem os dados a uma base comum, permitindo uma comparação entre os animais livres de tendências (Moreira \& Cardellino, 1994).

A idade do bezerro ao desmame (IDB) tem grande influência sobre o peso a desmama (PD) e o ganho de peso no período do nascimento ao desmame (GMD), observando-se o decréscimo do ganho, à medida que aumenta a idade do animal. A Beef Improvement Federation (BIF, 1996) recomenda usar pesos e ganhos de pesos ajustados, linearmente, para a idade do bezerro (períodos de 90 dias). Porém, diversos trabalhos (Paz, 1997; Pelicioni, 2000) indicam que o ajuste linear padrão (205 dias de idade) para IDB não remove toda a variação resultante deste efeito no PD e GMD, mesmo dentro de intervalos de 90 dias, mostrando efeito não-linear de IDB sobre estas características.

Outros efeitos não genéticos que apresentam influências sobre o crescimento de bezerros até a desmama são o ano, mês e estação de nascimento. No entanto, recentemente, alguns autores como Paz (1997) e Bocchi (1999) recomendam o efeito de DJN e o cálculo de fatores de correção para este efeito, preferencialmente, a inclusão do mês de nascimento no modelo de análise. Porém, os efeitos ambientais que mais afetam o ganho em peso dos bezerros são aqueles associados às influências maternas. A idade da mãe afeta o peso do bezerro ao desmame, porque vacas mais novas tendem à menor produção de leite do que as vacas adultas (Giannoni \& Giannoni, 1983). O efeito da idade da vaca pode ser observado, uma vez que o peso dos bezerros à desmama tende a aumentar com a IDV, até esta atingir a maturidade fisiológica. Após a maturidade, a tendência é de redução no peso dos bezerros. A determinação dos fatores de correção para IDV deve ser feita para machos e fêmeas separadamente, pois existem diferenças relevantes entre os dois sexos, quanto aos pesos.

A maioria dos trabalhos em que se estudou o efeito da idade da vaca ao parto sobre as características de crescimento no período pré-desmama utilizou como opção de modelagem os polinômios ordinários, observando-se a existência de efeito quadrático. No entanto, alguns autores têm mostrado a existência do efeito quadrático-quadrático, mediante a modelagem por polinômios segmentados (Campos et al., 1989; Lucero et al., 1995).

Para o ajuste dos pesos em gado de corte, a BIF (1996) recomenda fatores de correção aditivos, para as condições dos Estados Unidos, onde a variação dos registros de produção é pequena. Porém, a utilização de fatores multiplicativos, quando se trabalha com grandes variações nas produções dos rebanhos, apresenta vícios menores, quando comparados aos fatores aditivos. Por este motivo, Bocchi (1999) e Teixeira et al. (1999) justificaram a utilização dos fatores de correção multiplicativos para corrigir dados relacionados ao rebanho de corte brasileiro, uma vez que os valores observados de ganho médio diário de peso, variaram muito.

Objetivou-se com este trabalho:(1)estudara influência da idade da vaca ao parto (IDV) e da data juliana de nascimento (DJN) sobre o peso a desmama (PD) e ganho médio diário de peso (GMD) no período pré-desmama de bezerros Gir e (2) determinar fatores de correção para estes efeitos (IDV e DJN) sobre PD e GMD.

\section{Material e Métodos}

Os dados utilizados, no presente trabalho, são referentes a 18.520 registros de animais da raça Gir, nascidos no período de 1976 a 1994, obtidos do arquivo da ABCZ (Associação Brasileira dos Criadores de Zebu). Do arquivo geral da $\mathrm{ABCZ}$, foi desenvolvido um segundo arquivo contendo somente os dados até a desmama.

Foram utilizados, nas análises, dados de peso à desmama (PD) e ganho médio diário pré-desmama (GMD) de animais criados em diversas condições no Brasil. As pesagens foram realizadas a cada três meses, e todos os dados disponíveis foram utilizados para o cálculo do ganho médio diário do nascimento a desmama. PD e GMD foram pré-ajustados para idade do bezerro, usando os fatores de correção estimados pela ABCZ (1996).

Foram consideradas as variáveis, idade da vaca ao parto (IDV) e data juliana de nascimento (DJN), sendo que a IDV variou de 3 a 17 anos e a DJN oscilou de 1 a 365 dias.

O grupo contemporâneo (GC) foi definido pelo ano de nascimento, estação de nascimento, fazenda (Estado), criador (município), sexo, categoria e regime alimentar 
(1 - pasto; 2 - semi-confinamento e 3 - confinamento). Como muitos animais não permaneceram em apenas um sistema, foi considerado o maior valor do escore associado ao regime alimentar pelo qual o animal tenha passado, conforme sistema oficial da ABCZ.

Os animais cujas mães apresentavam idades inferiores a três anos e maiores de 17 anos foram eliminados, devido ao número restrito de observações, assim como os grupos contemporâneos com menos de cinco observações. Após estas considerações, o arquivo apresentou 10.685 e 18.339 observações de PD e GMD, de animais pertencentes a 1.229 e 1.979 grupos contemporâneos (GC), respectivamente.

Depois de testada a significância dos efeitos de IDV e DJN, sobre PD e GMD, determinou-se o comportamento e a forma das curvas para os mesmos.

Para as fêmeas, as curvas de PD e GMD, em função de IDV, foram ajustadas utilizando-se o Polinômio Segmentado Quadrático-Quadrático, com 'nó', ou ponto de junção aos 3,8 anos de idade, de acordo com o seguinte modelo:

$$
\hat{Y} x=a_{0}+a_{1} x+a_{2} x^{2}+\left(b_{2}-a_{2}\right) z
$$

em que $\mathrm{z}=0$, se $\mathrm{x} \leq \mathrm{k} \quad \mathrm{e} \mathrm{z}=(\mathrm{x}-\mathrm{k})^{2}$, se $\mathrm{x}>\mathrm{k}$; $\mathrm{x}=$ estimativa do peso à desmama ou ganho médio diário de peso pré-desmama, em $\mathrm{kg} ; \mathrm{x}=$ idade da vaca (IDV), em anos; $a_{0}, a_{1}, a_{2}, e b_{2}=$ parâmetros da função; e $\mathrm{k}=$ "nó" ou ponto de junção entre os segmentos do polinômio.

Para os machos, as curvas de PD e GMD em função de IDV foram ajustadas utilizando-se o Polinômio Segmentado Quadrático-QuadráticoQuadrático, com "nós", ou pontos de junção aos 4,1; 12,7 e 4,0;8,2 anos de idade, respectivamente, de acordo com o seguinte modelo:

$$
\hat{Y} x=a_{0}+a_{1} x+a_{2} x^{2}+\left(b_{2}-a_{2}\right) z_{1}+\left(c_{2}-b_{2}\right) z_{2}
$$
em que $\mathrm{z}_{1}=0$, se $\mathrm{x} \leq \mathrm{k}_{1}$ e $\mathrm{z}_{1}=\left(\mathrm{x}-\mathrm{k}_{1}\right)^{2}$, se $\mathrm{x}>\mathrm{k}_{1}$ $\mathrm{z}_{2}=0$, se $\mathrm{x} \leq \mathrm{k}_{2}$ e $\mathrm{z}_{2}=\left(\mathrm{x}-\mathrm{k}_{2}\right)^{2}$, se $\mathrm{x}>\mathrm{k}_{2} ; \mathrm{a}_{0}, \mathrm{a}_{1}, \mathrm{a}_{2}$, $\mathrm{b}_{2}$ e $\mathrm{c}_{2}=$ parâmetros da função; e $\mathrm{k}_{1}$ e $\mathrm{k}_{2}=$ "nós" ou pontos de junção entre os segmentos do polinômio.

As curvas de PD e GMD, em função de DJN, foram ajustadas utilizando-se Polinômio Segmentado Quadrático-Quadrático, com 'nós' ou pontos de junção aos 126 e 167 dias, respectivamente, de acordo com o seguinte modelo:

$$
\hat{Y} x=a_{0}+a_{1} x+a_{2} x^{2}+\left(b_{2}-a_{2}\right) z
$$

em que $z=0$, se $x \leq k$ e $z=(x-k)^{2}$, se $x>k$

$\mathrm{O}$ ajuste das curvas referentes aos polinômios segmentados foi realizado pelo método de quadrados mínimos, utilizando-se o procedimento REG do SAS (SAS, 1998).
A determinação dos polinômios segmentados e a escolha dos "nós" iniciais foram feitas após a inspeção visual dos pontos nos gráficos de médias observadas de PD e GMD, em função de IDV e DJN. A seguir, foram realizadas análises sucessivas utilizando-se os 10.685 dados de PD e os 18.339 dados do arquivo de GMD, fazendo-se variar o "nó" inicialmente escolhido, com o objetivo de encontrar o "nó" ou ponto de junção mais adequado, o qual foi determinado quando a análise apresentou a menor soma de quadrados de resíduo, procedendo-se, então, à estimativa dos parâmetros do modelo.

A definição dos polinômios deu-se após o ajuste das curvas dos efeitos em estudo (IDV, DJN) sobre PD e GMD.

Os fatores de correção de PD e GMD para IDV e DJN foram calculados utilizando-se as soluções obtidas para cada efeito, no ajuste dos polinômios segmentados, sendo:

$$
\mathrm{FC}_{\mathrm{X}}=\mathrm{D}_{\text {base }} / \mathrm{D}_{\mathrm{X}}
$$

em que $\mathrm{FC}_{\mathrm{X}}=$ fator de correção para o ponto $\mathrm{X}$ em função da base; $D_{\text {base }}=$ peso a desmama ou ganho médio diário de peso pré-desmama, estimado na base; e $\mathrm{D}_{\mathrm{X}}=$ peso à desmama ou ganho médio diário de peso pré-desmama, estimado no ponto $\mathrm{X}$.

Para o cálculo dos fatores de correção foram tomados, como base, os valores de 7,0 anos para IDV, tanto para machos quanto para fêmeas, representando a idade com melhores médias de PD e GMD e 45, 135, 225 e 315 dias para DJN, representando as médias de dias em cada estação dentro do calendário juliano. Os valores de PD e GMD, estimados nestas bases, foram 139,85 e 0,5722 kg, para machos, e 121,11 e $0,4804 \mathrm{~kg}$, para fêmeas, em função de IDV, respectivamente, e 120,65; 121,76; 130,74; e 133,66 kg para PD e 0,4722; 0,4571; 0,5153; e 0,5315 kg para GMD, em função de DJN.

\section{Resultados e Discussão}

As médias observadas de PD e GMD, previamente corrigidas para idade do bezerro, foram, respectivamente, $124,49 \mathrm{~kg}$, variando de 44,00 a $240,00 \mathrm{~kg}$, e $0,5085 \mathrm{~kg} / \mathrm{dia}$, variando de 0,1036 a $1,2379 \mathrm{~kg} /$ dia. Estes valores indicam que a raça Gir pode ser considerada uma raça leve, provavelmente, em razão de a seleção do Gir ser direcionada não somente para a produção de carne, mas também para a produção de leite. 
Efeito da idade da vaca

O PD observado variou de 53,00 a 240,00 kg para machos e de 44,00 a $229 \mathrm{~kg}$ para as fêmeas. Já o GMD variou de 0,117 a 1,238 kg/dia para machos e 0,104 a $1,227 \mathrm{~kg} /$ dia para fêmeas e a idade da vaca ao parto (IDV) variou de 3 a 17 anos.

A IDV, para os machos, foi modelada como polinômio segmentado quadrático-quadrático- quadrático com 'nós' aos 4,1 e 12,7 anos para PD e 4,0 e 8,2 anos para GMD. Para as fêmeas, a IDV foi modelada como polinômio segmentado quadráticoquadrático com 'nó' aos 3,8 anos, para as duas características.

Na Tabela 1 são apresentadas as estimativas dos parâmetros para o efeito de IDV sobre PD e GMD, utilizando-se os polinômios segmentados.

Tabela 1 - Estimativas dos parâmetros para o efeito de idade da vaca ao parto (IDV) sobre o peso à desmama e a média de ganho diário pré-desmama, para machos e fêmeas, utilizando-se o polinômio segmentado

Table 1 - Parameter estimates for age of cow at calving (AC) on weaning weight and average daily gain, for males and females by segmented polynomial regression

\begin{tabular}{|c|c|c|c|c|}
\hline \multicolumn{5}{|c|}{$\begin{array}{c}\text { Peso à desmama } \\
\text { Weaning weight }\end{array}$} \\
\hline \multicolumn{5}{|c|}{ Machos (Males) } \\
\hline $\begin{array}{l}\mathrm{IDV} \\
A C\end{array}$ & $\begin{array}{l}\text { Parâmetro estimado } \\
\text { Estimated parameter }\end{array}$ & $\begin{array}{l}\text { Erro-padrão } \\
\text { Standard error }\end{array}$ & $\begin{array}{l}\text { t para } \mathrm{H}_{0} \\
\text { t for } H_{0}\end{array}$ & Prob $>|t|$ \\
\hline \multicolumn{5}{|l|}{ Intercepto } \\
\hline Intercept & 316,3979 & 51,4013 & 6,155 & 0,0001 \\
\hline $\mathrm{X}_{1}$ & $-95,1378$ & 25,5378 & $-3,725$ & 0,0002 \\
\hline$X_{2}^{1}$ & 11,9020 & 3,1434 & 3,786 & 0,0002 \\
\hline $\mathrm{Z}_{1}^{2}$ & $-12,2214$ & 3,1662 & $-3,860$ & 0,0001 \\
\hline $\mathrm{Z}_{2}^{1}$ & 1,1363 & 0,3214 & 3,535 & 0,0004 \\
\hline $\mathrm{SQE}=3966324,233$ & \multicolumn{2}{|c|}{$\mathrm{R}^{2}=1,19 \%$} & \multicolumn{2}{|c|}{$\mathrm{CV}=20,94 \%$} \\
\hline \multicolumn{5}{|c|}{ Fêmeas (Females) } \\
\hline \multicolumn{5}{|l|}{ Intercepto } \\
\hline Intercept & 447,5932 & 84,6729 & 5,285 & 0,0001 \\
\hline $\mathrm{X}_{1}$ & $-173,7675$ & 44,8912 & $-3,871$ & 0,0001 \\
\hline$X_{2}^{1}$ & 22,9874 & 5,9254 & 3,879 & 0,0001 \\
\hline$Z_{1}^{2}$ & $-23,0954$ & 5,9362 & $-3,891$ & 0,0001 \\
\hline $\mathrm{SQE}=3239013,7149$ & \multicolumn{2}{|c|}{$\mathrm{R}^{2}=0,54 \%$} & \multicolumn{2}{|c|}{$\mathrm{CV}=20,74 \%$} \\
\hline
\end{tabular}

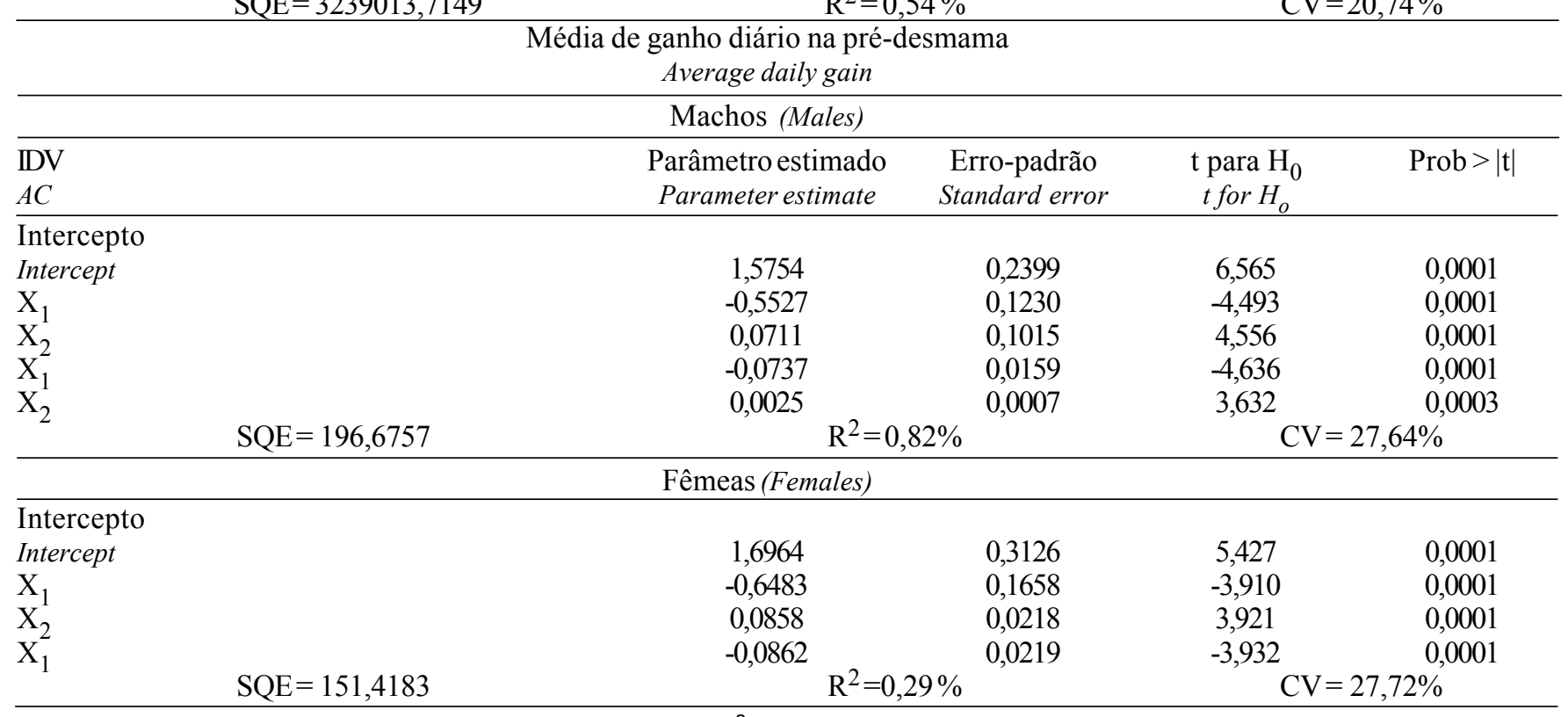

SQE (SSE) = soma de quadrado de resíduo (error sum of square); $\mathrm{R}^{2}=$ coeficiente de determinação (coefficient of determination); CV = coeficiente de variação (coefficient of variation); $\mathrm{t}$ = estatística $\mathrm{T}$ de Student $(T$ statistic of Student). 
As equações estimadas para o efeito de IDV sobre PD e GMD de machos e fêmeas, foram:

PD:

$\hat{\mathrm{Y}}_{\text {IDV }}($ machos $)=316,3979-95,1378 \mathrm{X}$ $+11,9020 X^{2}-12,2214 Z_{1}+1,1363 Z_{2}$

$Z_{1}=0$, se $x \leq 4,1$ e $Z_{1}=(x-4,1)^{2}$, se $x>4,1$

$Z_{2}=0$, se $x \leq 12,7$ e $Z_{2}=(x-12,7)^{2}$, se $x>12,7$

$\hat{\mathrm{Y}}_{\mathrm{IDV}}$ (fêmeas) $=447,5932-173,7675 \mathrm{X}+$ $22,9874 X^{2}-23,0954 Z$

$Z=0$, se $x \leq 3,8$ e $Z=(x-3,8)^{2}$, se $x>3,8$

GMD:

$\hat{\mathrm{Y}}_{\text {IDV }}($ machos $)=1,575461-0,552723 \mathrm{X}+$ $0,07114 X^{2}-0,073731 Z_{1}+0,002561 Z_{2}$

$Z_{1}=0$, se $x \leq 4,0$ e $Z_{1}=(x-4,0)^{2}$, se $x>4,0$

$Z_{2}=0$, se $x \leq 8,2$ e $Z_{2}=(x-8,2)^{2}$, se $x>8,2$

$\hat{\mathrm{Y}}_{\text {IDV }}$ (fêmeas) $=1,696442-0,648393 \mathrm{X}+$ $0,085832 X^{2}-0,086237 Z$

$Z=0$, se $x \leq 3,8$ e $Z=(x-3,8)^{2}$, se $x>3,8$

em que:

$\hat{Y}=$ peso à desmama ou ganho médio diário prédesmame predito

$\mathrm{X}=$ idade da vaca ao parto.

As curvas ajustadas pelos polinômios segmentados para PD e GMD preditos em função de IDV estão apresentadas nas Figuras 1 e 2, para machos e fêmeas, respectivamente.

As curvas apresentadas nas Figuras 1 e 2 evidenciaram a existência da interação entre os efeitos de sexo e idade da vaca ao parto. Os animais nascidos de novilhas (3 anos), tanto machos quanto fêmeas, apresentaram maiores PD e GMD, com suave declínio no ano seguinte. A partir dos 4 anos de idade da vaca, os bezerros (machos ou fêmeas) mostraram tendências crescentes de $\mathrm{PD}$ e GMD, à medida que a mãe foi atingindo a maturidade, e, após apresentarem um platô, o PD e GMD das fêmeas e GMD dos machos passaram a declinar com o envelhecimento da vaca. Já o PD para os machos apresentou crescimento acentuado dos 14 aos 17 anos de idade da vaca ao parto. Estes comportamentos, provavelmente, estiveram relacionados com a produção de leite da vaca, em função da sua idade ao parto, sendo, assim, dependentes das atividades fisiológicas que ocorreram no animal durante sua vida produtiva. Além disso, provavelmente, a observação da influência diversificada deste efeito para machos e
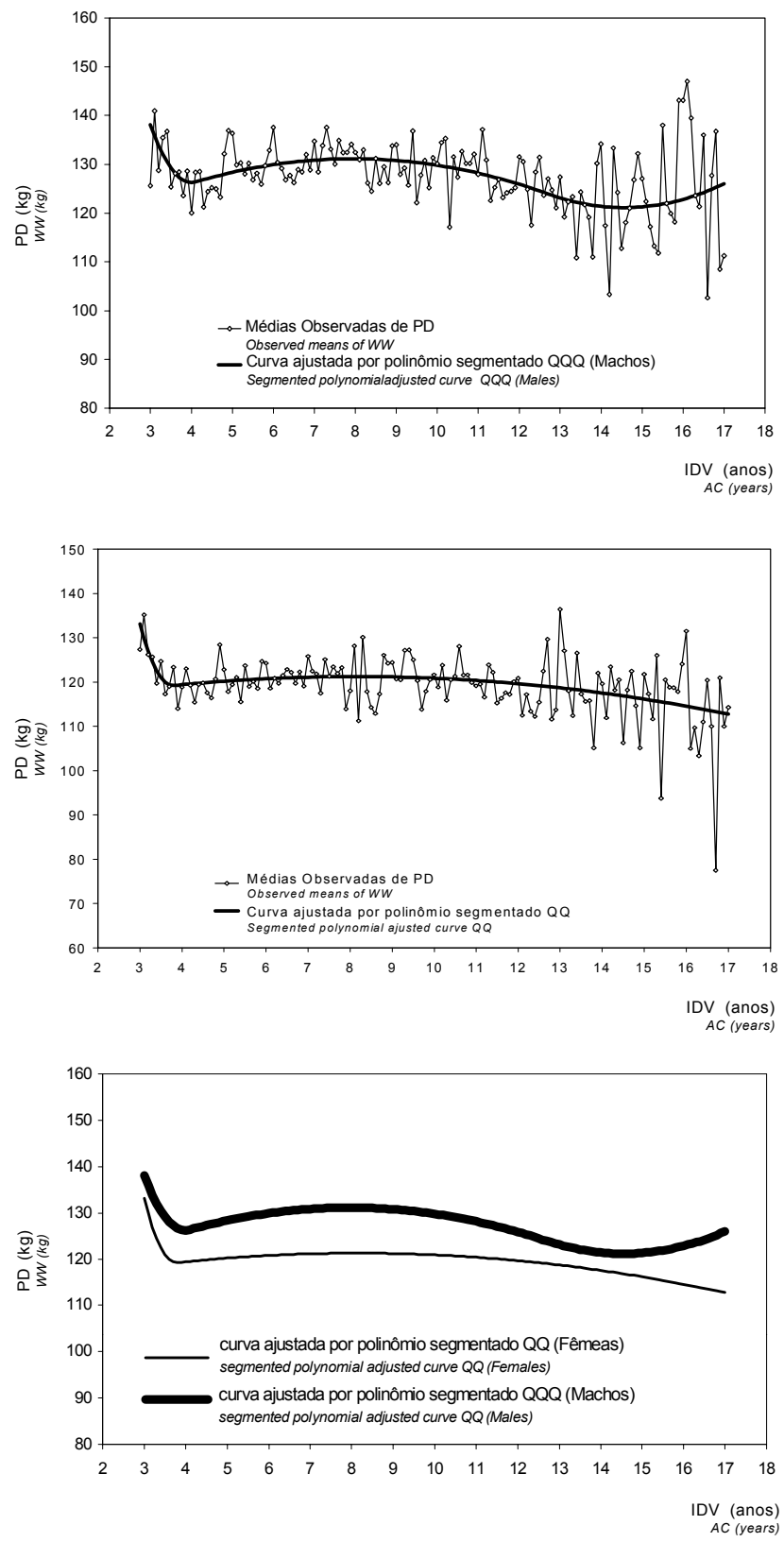

Figura 1 - Médias observadas e curvas ajustadas pelos polinômios segmentados quadráticoquadrático-quadrático (QQQ) para o peso a desmama (PD), em kg, de bezerros machos e fêmeas da raça Gir, por idade da vaca ao parto (IDV), em anos.

Figure 1 - Observed means and adjusted curves fitted by quadratic-quadratic-quadratic segmented polynomial regression $(Q Q Q)$ to weaning weight (WW), in $\mathrm{kg}$, of Gyr males and females calves, according to age of cow at calving $(A C)$, in years. 

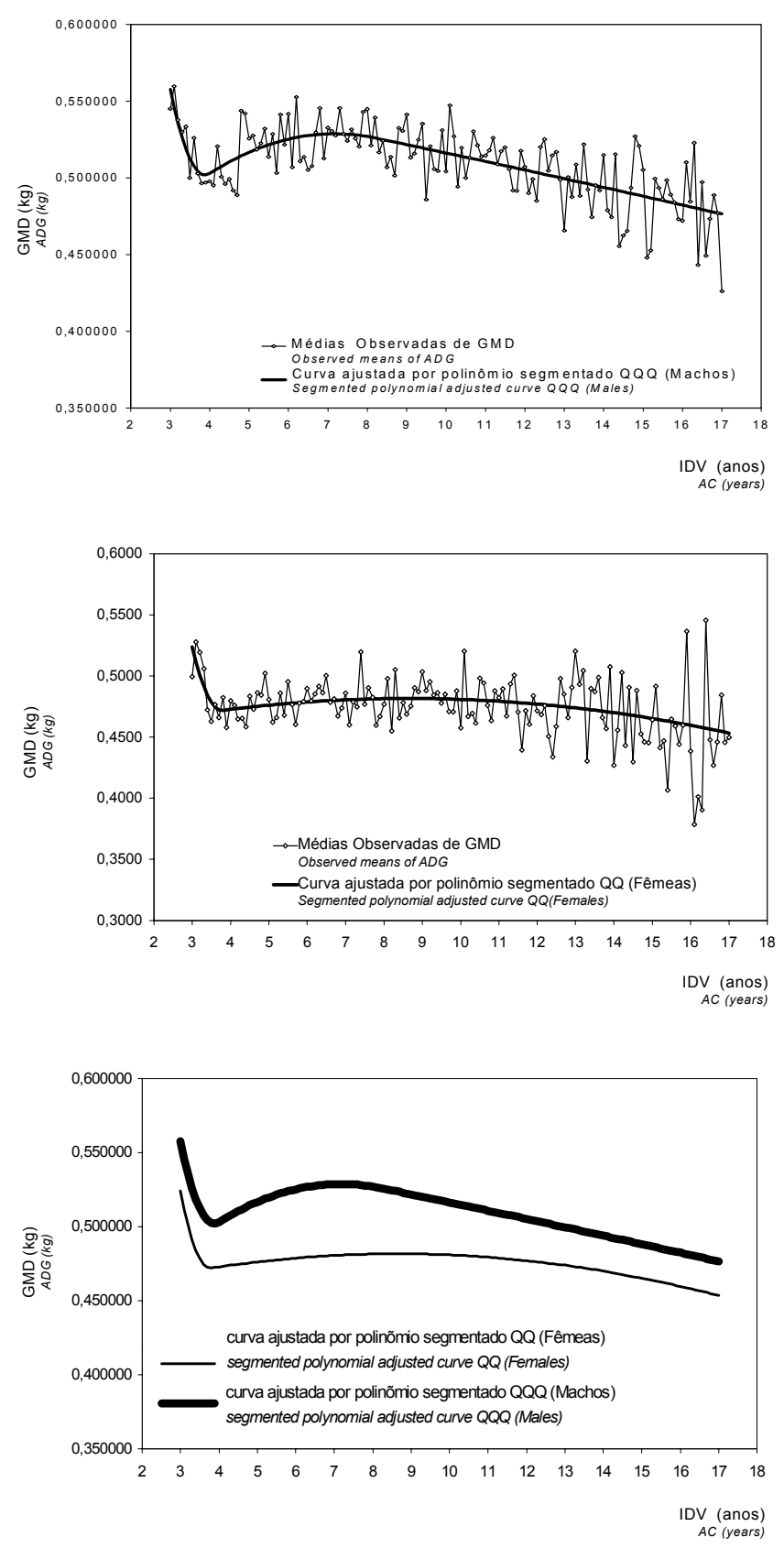

Figura 2 - Médias observadas e curvas ajustadas pelos polinômios segmentados quadrático-quadrático (QQ) para a média de ganho diário na prédesmama (GMD), em kg, de bezerros machos e fêmeas da raça Gir, por idade da vaca ao parto (IDV), em anos.

Figure 2 - Observed means and adjusted curve fitted by quadraticquadratic segmented polynomial regression (QQ) to preweaning average daily gain (ADG), in $\mathrm{kg}$, of $\mathrm{Gyr}$ males and females calves, according to age of cow at calving $(A C)$, in years. fêmeas poderia, também, ser atribuída a uma diferença no potencial do seu crescimento. Os machos, possivelmente, aproveitariam melhor a produção de leite de suas mães, com especial atenção para o seu pico de produção. O desempenho dos animais, tanto para PD como para GMD atingiu o pico por volta dos 7,5 anos de idade da vaca ao parto, tanto para machos quanto para fêmeas. Entretanto, os machos apresentaram aumento de peso e ganho pré-desmame dos 4 aos 8 anos de idade, mais acentuado que nas fêmeas. Praticamente, PD e GMD mostraram-se estáveis dos 4 aos 11 anos de IDV para fêmeas e, na seqüência, apresentaram leve declínio na curva ajustada. Porém, os dados observados na curva de fêmeas apresentaram-se bastante dispersos a partir dos 11 anos de IDV, podendo-se verificar que, a partir deste ponto, o ajuste acompanhou a tendência da curva.

No final da curva, para PD, evidenciou-se muita distinção para machos e fêmeas. Na Figura 1, constatase ligeiro aumento no PD dos machos, após os 13 anos, enquanto, para as fêmeas, observa-se declínio constante de PD. Como a intensidade da seleção de machos é maior do que a de fêmeas, a diferenciação do manejo poderia compensar possível desvantagem desse grupo, para a obtenção dos registros.

A existência da diferença na média do ganho diário entre os dois sexos, com vantagem para os machos (Figuras 1 e 2), também, foi observada por outros autores. Paz (1997), trabalhando com Nelore, relatou GMD predito que variou de 0,65293 a $0,72476 \mathrm{~kg} /$ dia para machos e 0,58220 a $0,64101 \mathrm{~kg} / \mathrm{dia}$ para fêmeas e, para compensar essa diferença, recomendou que as novilhas, por apresentarem maior desgaste para criar seus bezerros, poderiam ser manejadas de maneira diferenciada, dependendo do sexo de seus filhos. Novilhas, mães de machos, poderiam receber suplementação alimentar na tentativa de compensar este maior desgaste.

Teixeira et al. (1999) observaram, em animais cruzados, que os machos, além de apresentarem maior GMD, foram mais influenciados pela IDV, uma vez que as fêmeas apresentaram uma curva com pico menos evidente. Bocchi (1999), estudando quatro raças zebuínas: Nelore, Guzerá, Tabapuã e Indubrasil, verificou que o GMD das fêmeas e o pico de produção das vacas que desmamaram bezerras foram menores que para os machos, sendo, também, observado que a curva para fêmeas não apresentou pico 
evidente quanto para os machos, formando uma curva mais achatada.

Neste estudo, as vacas da raça Gir, a partir dos 12 anos de idade começaram a produzir bezerros mais leves que as novilhas. Essa tendência, também, foi observada por Bocchi (1999) com a raça Guzerá aos 9 anos, com a Tabapuã aos 11 anos, com a Indubrasil aos 12 anos, e com a Nelore, em torno dos 15 anos. Estes resultados mostraram que não é satisfatório manter no rebanho, vacas depois de determinada idade. Neste caso, o descarte destes animais poderia ser recomendado como estratégia para deixar pastagens mais disponíveis para as novilhas, possibilitando melhorias no manejo dessa categoria, o que permitiria melhor incremento na eficiência reprodutiva.

As curvas apresentadas nas Figuras 1 e 2 evidenciaram um comportamento diferenciado de novilhas dos 3 aos 3,5 anos, tanto para os machos quanto para as fêmeas. Nesta idade, foram constatadas taxas de ganho de peso de seus filhos, relativamente altas, uma vez que, no início da vida produtiva, a eficiência em ganho das crias costuma ser inferior. Este comportamento pode estar descrevendo um manejo diferenciado para as novilhas, uma vez que se trata de uma categoria que se encontra em crescimento e, ainda, amamentando sua cria, portanto, com maiores requerimentos nutricionais. Um manejo diferenciado dessas novilhas possibilitaria aos bezerros apresentarem maiores taxas de ganho de peso nesse período, o que pode ser a explicação para o observado neste estudo.

Os fatores de correção para o efeito de IDV sobre PD e GMD (Figura 3) variaram, respectivamente, de 0,94750 a 1,08033 e de 0,91714 a 1,07689 , para machos e, 0,90937 a 1,07415 e 0,96055 a 1,14007 , para fêmeas, mostrando diferenças de até $17,4 \%$ nos fatores de correção para IDV, em machos e de até $18,6 \%$, em fêmeas. Os fatores de ajuste para IDV de machos e fêmeas foram calculados considerando-se, como base, sete anos de idade da vaca. Este valor foi adotado por representar os melhores desempenhos dos bezerros para PD e GMD.

Observou-se na Figura 3, para machos e fêmeas, aumento dos fatores de correção estimados dos 3 aos 4 anos e decréscimo dos 4 aos 8 anos de idade da vaca, visando compensar o aumento de PD e GMD neste intervalo, no qual ocorreu o ganho máximo. Dos 8 aos 17 anos, verificou-se o aumento dos fatores de correção para fêmeas em PD e fêmeas e machos em GMD, proporcionados pela
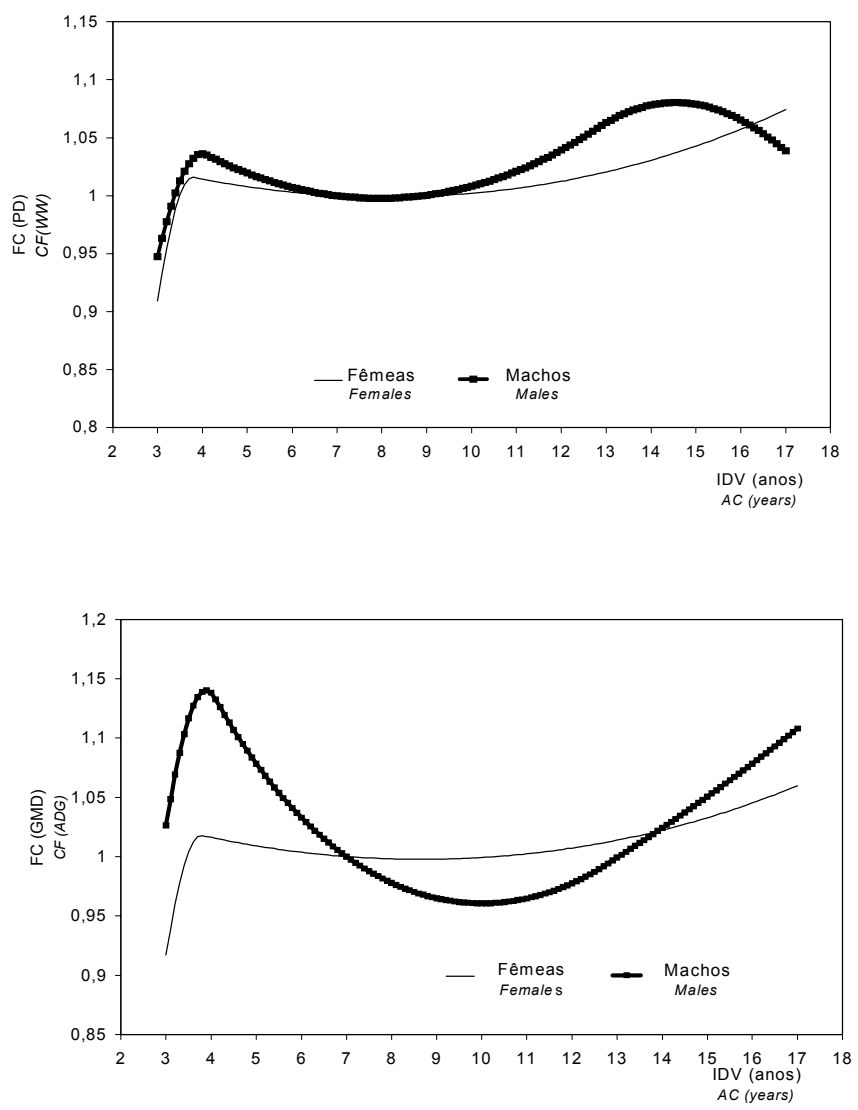

Figura 3 - Fatores de correção (FC) do peso à desmama (PD) e média de ganho diário pré-desmama (GMD) para o efeito da idade da vaca (IDV), em anos, para bezerros, machos e fêmeas, da raça Gir.

Figure 3 - Correction factors (CF) of weaning weight (WW) and preweaning average daily gain (ADG) for age of cow at calving (AC), in years, for male and female Gyr calves.

diminuição de PD e GMD. Já para machos, verificaram-se aumento dos valores de PD dos 8 aos 14 e ligeiro decréscimo a partir desta idade.

Efeito da data juliana de nascimento

A Figura 4 mostra as médias observadas de PD e GMD, em função de DJN e as curvas ajustadas pelos polinômios segmentados.

Observou-se tendência quadrática-quadrática de DJN sobre PD e GMD de bezerros Gir. Assim, a data de nascimento, dentro do calendário juliano, foi modelada como polinômio segmentado quadráticoquadrático, ou seja, uma função composta por dois segmentos quadráticos, com "nós", ou pontos de junção estimados aos 126 e 167 dias, para PD e GMD, respectivamente. Constam da Tabela 2 as estimativas dos parâmetros para o efeito de DJN sobre PD e 
Tabela 2 - Estimativas de parâmetros para o efeito da data juliana de nascimento (DJN) sobre o peso à desmama e média de ganho diário pré-desmama, utilizando-se polinômio segmentado.

Table 2 - Parameter estimates for julian date of birth (JDB) on weaning weight and preweaning average daily gain, by segmented polynomial regression

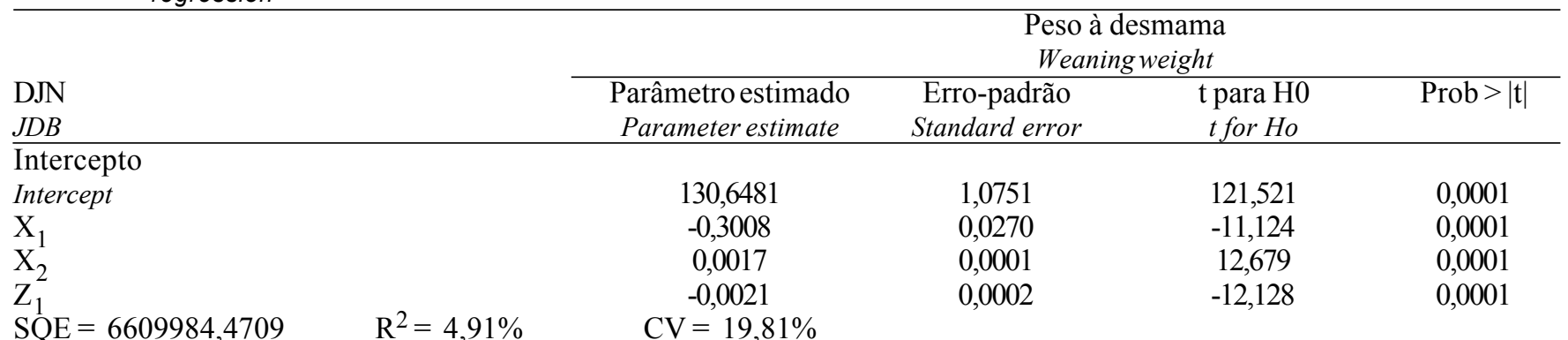

$\mathrm{SQE}=6609984,4709 \quad \mathrm{R}^{2}=4,91 \% \quad \mathrm{CV}=19,81 \%$

Ganho médio diário na pré-desmama

Preweaning average daily gain

Intercepto

Intercept

$\mathrm{X}_{1}$

$\mathrm{X}_{2}$

$\mathrm{Z}_{1}$

$$
\begin{array}{r}
0,516969 \\
-0,001318 \\
0,000006
\end{array}
$$

$\mathrm{SQE}=339,5731 \quad \mathrm{R}^{2}=4,72 \% \quad \mathrm{CV}=27,36 \%$

$\begin{array}{ccc}0,003915 & 130,498 & 0,0001 \\ 0,0000812 & -16,229 & 0,0001 \\ 0,0000003 & 18,880 & 0,0001 \\ 0,0000005 & -17,267 & 0,0001\end{array}$

SQE (SSE) = soma de quadrado de resíduo (error sum of square); $\mathrm{R}^{2}$ = coeficiente de determinação (coefficient of determination); $\mathrm{t}=$ estatística $\mathrm{T}$ de Student ( $T$ statistic of Student).
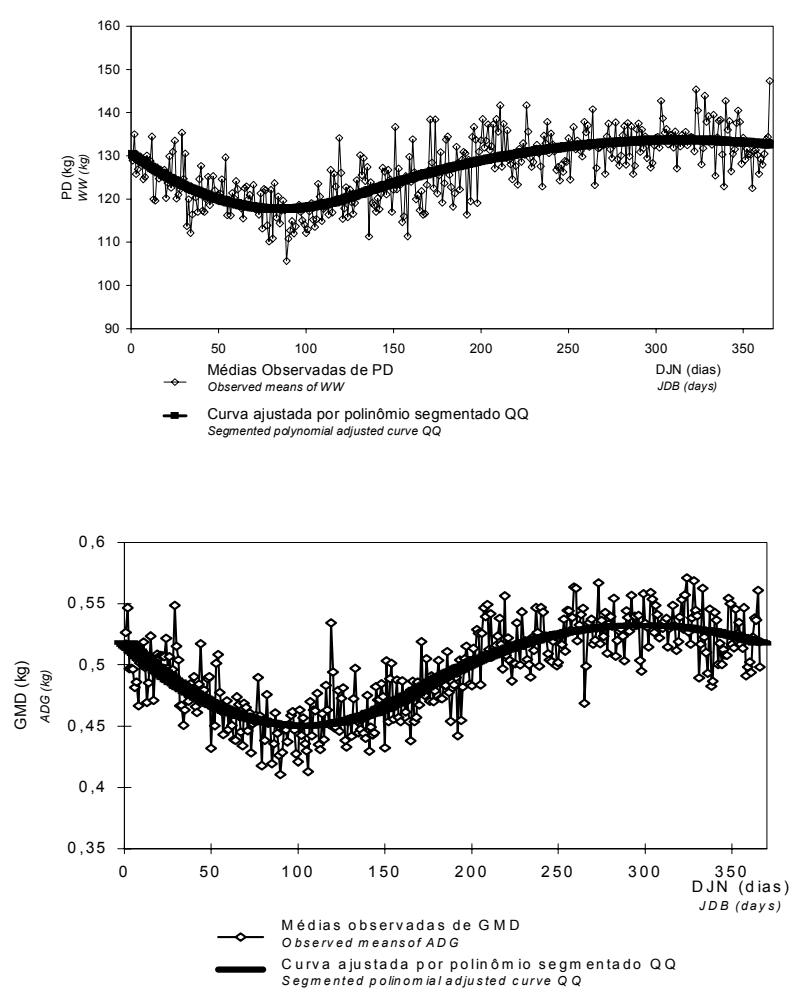

Figura 4 - Médias observadas e curvas ajustadas por polinômios segmentados quadrático-quadrático para peso à desmama e média de ganho diário pré-desmama (GMD), em kg, de bezerros da raça Gir, por data juliana de nascimento (DJN), em dias.

Figure 4 - Observed means and adjusted curves fitted by quadratic-quadratic segmented polynomial regression (QQ) to weaning weight and preweaning average daily gain (ADG), in $\mathrm{kg}$, of Gyr calves, according to julian date of birth (JDB), in days.
GMD, utilizando-se Polinômios Segmentados.

As equações estimadas para o efeito de DJN sobre PD e GMD foram:

$$
\hat{\mathrm{Y}}_{\mathrm{PD}}=130,648110-0,300841 \mathrm{X}+0,001750 \mathrm{X}^{2}-
$$

sendo: $Z=0$, se $X \leq 126$ e $Z=(X-126)^{2}$, se $X>126$

$$
\begin{gathered}
\hat{\mathrm{Y}}_{\mathrm{GMD}}=0,516969-0,001318 \mathrm{X}+0,0000061 \mathrm{X}^{2} \\
-0,000009 \mathrm{Z}
\end{gathered}
$$

sendo: $Z=0$, se $X \leq 167$ e $Z=(X-167)^{2}$, se $X>167$ em que:

$\hat{\mathrm{Y}}=$ média de ganho diário pré-desmama predito;

$\mathrm{X}=$ data juliana de nascimento.

Para o cálculo dos valores preditos de PD e GMD em cada estação utilizou-se como valor base a média de cada estação, uma vez que procurou-se manter as diferenças entre as estações.

OPD, em cada estação do ano, variou de 66 a $194 \mathrm{~kg}$, com média de 122,20 kg no verão; de 66 a 197 kg, com média de 121,54 kg no outono; de 66 a $194 \mathrm{~kg}$, com média de 130,98 kg no inverno; e de 67 a 194 kg, com média de 133,42 kg na primavera. Já o GMD, variou de 0,104 a $1,128 \mathrm{~kg} /$ dia com média de $0,502 \mathrm{~kg} /$ dia no verão; de 0,459 a $1,238 \mathrm{~kg} / \mathrm{dia}$, com média de $0,450 \mathrm{~kg} / \mathrm{dia}$ no outono; de 0,118 a 1,208 kg/dia, com média de $0,512 \mathrm{~kg} /$ dia no inverno; e de 0,117 a $1,227 \mathrm{~kg} /$ dia, com média de $0,538 \mathrm{~kg} /$ dia na primavera. 
As curvas apresentaram tendência decrescente do $1^{\circ}$ dia até, em torno, do $105^{\circ}$ dia. A partir daí, passaram a crescer, atingindo o máximo, por volta, do $275^{\circ}$ dia, formando um pequeno patamar que se estendeu até o $330^{\circ}$ dia. A partir deste ponto, demonstraram ligeiro decréscimo até o $365^{\circ}$ dia.

A Figura 4 evidenciou que os animais nascidos no início da primavera $\left(265^{\circ}\right.$ a $315^{\circ}$ dia $)$ apresentaram melhor desempenho para PD e GMD. Uma possível explicação para este fato é que suas mães, devido à maior oferta de alimento, tiveram maior produção de leite e, portanto, maior disponibilidade de alimento para suas crias. Analisando este fato, percebe-se a importância de estabelecer-se uma estação reprodutiva. Uma estação de monta entre o $360^{\circ}$ e $60^{\circ}$ dia (dezembro a fevereiro) resultaria em bezerros mais eficientes em ganhar peso até a desmama, possibilitando a obtenção de um produto mais pesado com conseqüente incremento no sistema da produção de carne.

Os fatores de correção para o efeito da data juliana de nascimento estão representados na Figura 5.

Os fatores de correção para DJN foram estimados dentro da estação, para que o efeito da mesma fosse mantido, permitindo maior flexibilidade e planejamento correto do melhor período a ser realizada a estação de monta. Para a raça Gir estes valores variaram de 0,9256 no $1^{\circ}$ dia a 1,0340 no $93^{\circ}$ dia para PD e de 0,9112 no $1^{\circ}$ dia a 1,0551 no $181^{\circ}$ dia para GMD. Paz (1997) encontrou variação maior para os fatores de correção, em Nelore, sendo estes de 0,7425 aos $230^{\circ}$ / $239^{\circ}$ dia do calendário juliano a 1,3907 aos $300^{\circ} / 309^{\circ}$ dia. Fries (1996) e Bocchi (1999) observaram, também para a raça Nelore, respectivamente, variação de 0,9407 a 1,0512 e 0,9544 a 1,0344, que são semelhantes aos valores encontrados neste trabalho. Verificou-se, na Figura 5, aumento dos valores dos fatores de correção nos períodos de $1^{\circ}$ a $105^{\circ}$ dia e $310^{\circ}$ a $365^{\circ}$ dia do calendário juliano e, no intervalo de $106^{\circ}$ a $309^{\circ}$ dia, observou-se decréscimo dos fatores de correção, proporcionados pelos aumentos de PD e GMD, neste intervalo, demonstrando que os melhores desempenhos de PD e GMD seriam dos animais nascidos nas estações outono, inverno e parte da primavera.

Os resultados obtidos para PD e GMD, em função de IDV e DJN, permitiram constatar que as duas variáveis estudadas explicaram a mesma característica. Do ponto de vista da avaliação genética de animais, o GMD representa melhor ferramenta de trabalho, pois tem a vantagem de não embutir a seleção indireta para peso ao nascer como apresenta PD.
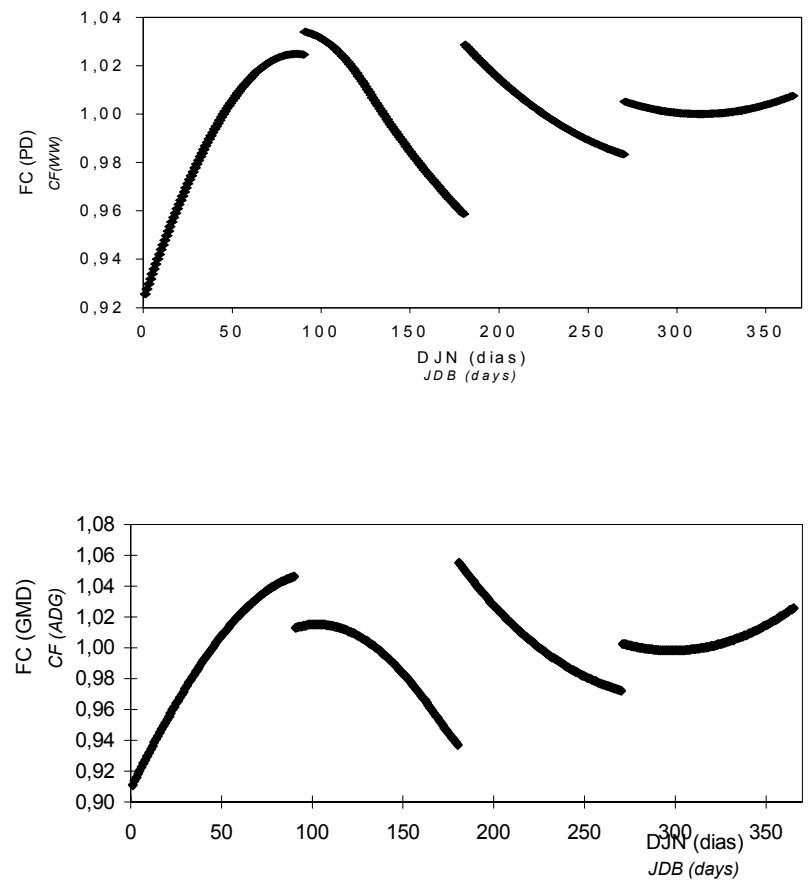

Figura 5 - Fatores de correção (FC) do peso à desmama (PD) e ganho médio diário pré-desmama (GMD) para o efeito de data juliana de nascimento (DJN), em dias, para bezerros da raça Gir.

Figure 5 - Correction factors (CF) of weaning weight (WW) and preweaning average daily gain (ADG) for julian date of birth (JDB), in days, of male and Gyr calves.

\section{Conclusões}

As variáveis, idade da vaca ao parto e data juliana de nascimento foram importantes fontes de variação sobre o peso à desmama e média de ganho diário na pré-desmama, devendo ser corrigidas.

A determinação de fatores de correção para a idade da vaca ao parto deve ser feita, separadamente, para machos e fêmeas.

A determinação e utilização de fatores de correção para a data juliana de nascimento devem levar em consideração as diferenças entre as estações do ano, de modo a permitir planejamento adequado da estação de monta/reprodução.

Recomenda-se manejo diferenciado às novilhas mães de bezerros machos, visando satisfazer maior necessidade de leite de suas crias, e planejamento de uma estação de monta no período de dezembro a fevereiro, visando à obtenção de bezerros mais pesados à desmama. 


\section{Literatura Citada}

ASSOCIAÇÃO BRASILEIRA DOS CRIADORES DE ZEBU ABCZ Gensys Consultores e Associados. CONSIST.FOR (Programa fonte que realiza consistência e ajustes para os dados da ABCZ).

BEEF IMPROVEMENT FEDERATION. Guidelines for uniform beef improvement programs. Raleigh: United State Department Agriculture - North Carolina State University, 1996. p.155.

BOCCHI, A.L. Efeito da idade da vaca e da data juliana de nascimento, sobre o ganho médio diário de bezerros de corte durante o período pré-desmame. Jaboticabal: Universidade Estadual Paulista, 1999. p.109. Trabalho (Graduação em Zootecnia) - Universidade Estadual Paulista, 1999.

CAMPOS, L.T.; SILVA, P.R.; FRIES, L.A. Fatores de correção para efeitos ambientais que afetam o ganho de peso do nascimento a desmama em bovinos da raça Nelore. Coletânea de pesquisas inéditas sobre Zebu. Uberaba: Empresa de Pesquisa Agropecuária de Minas Gerais, 1989. p.108-123.

FRIES, L.A. Efeito da data de nascimento sobre o peso à desmama em zebuínos. In: SIMPÓSIO NACIONAL DE MELHORAMENTO ANIMAL, 1., 1996, Ribeirão Preto. Anais... Ribeirão Preto: Sociedade Brasileira de Melhoramento Animal, 1996. p.255-256.

GIANNONI, M.A.; GIANNONI, M.L. Genética e melhoramento de rebanhos nos trópicos. São Paulo: Nobel, 1983. 463p.

LUCERO, C.E.; CAMPOS, L.T.; CARDOSO, F.F. et al. Fatores de correção do ganho de peso pré-desmame, pela idade da mãe e do bezerro, e a data de nascimento, na raça Nelore. In: REUNIÃO ANUAL DA SOCIEDADE BRASILEIRA DE ZOOTECNIA, 32., 1995, Brasília. Anais... Brasília: Sociedade Brasileira de Zootecnia, 1995. p.616-618.
MOREIRA, L.M.M.; CARDELLINO, R.A. Herdabilidade, repetibilidade e efeitos ambientais em bovinos Hereford. Pesquisa Agropecuária Brasileira, v.29, n.11, p.1795-1800.

PAZ, C.C. Efeitos ambientais e genéticos que afetam o ganho de peso pré-desmame em bovinos da raça Nelore. Jaboticabal: Universidade Estadual Paulista, 1997. 117p. Dissertação (Mestrado em Zootecnia) - Universidade Estadual Paulista, 1997.

PELICIONI, L.C. Polinômios segmentados no ajuste de idade e efeitos da linhagem citoplasmática para características de crescimento e produção de leite em bovinos da raça Caracu. Jaboticabal: Universidade Estadual Paulista, 2000. 112p. Dissertação (Mestrado em Zootecnia) - Universidade Estadual Paulista, 2000.

SAS INSTITUTE. SAS Language Guide, v.6. 11.ed. Care: 530p.

TEIXEIRA, R.A.; FRIES, L.A.; ALBUQUERQUE, L.G. Efeitos ambientais que afetam o ganho de peso pré-desmama em animais das raças Angus, Hereford, Nelore e produtos cruzados Angus x Nelore e Hereford x Nelore. In: REUNIÃO ANUAL DA SOCIEDADE BRASILEIRA DE ZOOTECNIA, 36., 1999, Porto Alegre. Anais... Porto Alegre: Sociedade Brasileira Brasileira, 1999. p.153. 